\title{
Innovación educativa: La experiencia de las carreras pedagógicas en la Universidad de Los Lagos, Chile
}

\section{CLAUDIA GONZÁLEZ CASTRO}

Universidad de Los Lagos - Chile

MARCELA CRUZAT ARRIAGADA*

Universidad de Los Lagos - Chile

Recibido el 31-07-18; primera evaluación el 09-08-19;

segunda evaluación el 13-08-19; aceptado el 28-08-19

\section{RESUMEN}

La innovación educativa es un objetivo relevante para las instituciones de educación superior, en especial ,para las instituciones formadora de profesores, por el impacto que tiene en el sistema educacional de un país. La Universidad de Los Lagos, universidad regional de la ciudad de Osorno en Chile, realizó una revisión del estado de la innovación educativa entre sus carreras pedagógicas. A través del análisis de entrevistas a académicos y la revisión de documentos institucionales, se concluyó que existen dificultades para delimitar el concepto de innovación; que la innovación en las carreras pedagógicas se inicia con la innovación curricular impulsada por el Modelo Educativo Institucional basado en competencias; y que las experiencias de innovación docente son, principalmente, innovaciones silenciosas.

Palabras clave: innovación educativa, Educación Superior, formación de profesores.

\footnotetext{
* Profesora General Básica, magíster en Educación y doctora en Cultura Latinoamericana. Profesional de la Unidad de Desarrollo Docente de la Universidad de Los Lagos, Osorno, Chile. Líneas de investigación: estudios de género, étnicos y latinoamericanos poscoloniales. Correo electrónico: claudia. gonzalez@ulagos.cl

** Licenciada en Educación, educadora de Párvulos y magíster en Ciencias de la Educación. Académica y jefe de la carrera Educación Parvularia en la Universidad de Los Lagos, Osorno, Chile. Línea de investigación: evaluación de aprendizaje. Correo electrónico: mcruzat@ulagos.cl
} 


\section{Educational Innovation: The experience of pedagogical careers at the University Of Los Lagos Chile}

\section{Abstract}

Educational innovation is a relevant aim for higher education institutions, especially for teacher training institutions, due to the impact on the educational system of a country. This study of educational innovation state, among their pedagogical careers, was made by Universidad de Los Lagos, regional university in the chilean city of Osorno. Through the analysis of academics interviews and the institutional documents review, it was concluded there are difficulties defining the innovation concept; that innovation in pedagogical careers starts with curricular innovation driven by the institutional model on competences-based education; and that the experiences of teaching innovation are, mainly, silent innovations.

Keywords: Educational innovation, Higher education, teacher training.

\section{Inovação educacional: A experiência de carreiras pedagógicas na Universidade de Los Lagos, Chile}

\section{RESUMO}

A inovação educacional é um objetivo relevante para as instituiçóes de ensino superior, especialmente para instituiçóes de formação de professores, devido ao impacto que tem no sistema educacional de um país. A Universidade de Los Lagos, universidade regional da cidade de Osorno, no Chile, fez este diagnóstico do estado de inovação educacional entre suas carreiras pedagógicas. Por meio da análise de entrevistas com acadêmicos e da revisão de documentos institucionais, concluiu-se que existem dificuldades na definição do conceito de inovação; que a inovação nas carreiras pedagógicas começa com a inovação curricular impulsionada pelo Modelo Educacional Institucional baseado em competências; e que as experiências de ensinar inovação são, principalmente, inovaçôes silenciosas.

Palavras-chave: inovação educacional, Educação superior, formação de professores 


\section{INTRODUCCIÓN}

El concepto de innovación aplicado a la educación refiere a un proceso complejo y sistémico de reflexión e intervención, en diferentes estamentos de una institución educacional, con el objetivo de mejorar el aprendizaje de los estudiantes. La multiplicidad de dimensiones y niveles de concreción dificultan su caracterización y estudio. Es imposible entender la innovación como un conjunto de pasos o procesos estables, sino más bien, como una respuesta de la institución educativa ante la transformación constante de la sociedad, la rápida obsolescencia del conocimiento y los problemas asociados a la educación superior. La innovación educativa se ha transformado en eje prioritario de organizaciones que promueven el desarrollo educativo, como United Nations Educational Scientific and Cultural Organization (Unesco), Organización de Estados Iberoamericanos (OEI), y la Organización para la Cooperación y Desarrollo Económico (OCDE), quienes ven en su multiplicidad de acciones, las posibles iniciativas para enfrentar los problemas propios de la educación en Chile y América Latina: inequidad en el acceso, deserción, desigualdad de calidad entre las instituciones, bajo desempeño en investigación y niveles de competencias entre egresados (OCDE, 2017, pp. 181-187).

El Consejo de Rectores de Universidades Chilenas (CRUCH) también ha declarado explícito interés en la innovación, como alternativa a la rigidez de los procesos educativos en Chile. Ha propiciado políticas nacionales para la renovación curricular en educación superior y el fortalecimiento de prácticas que contrarresten la deserción estudiantil y garanticen la calidad de la docencia entregada (Pey y Chauriye, 2011, p.6).

En el contexto de la Formación Inicial Docente (FID), la innovación educativa tiene especial relevancia. Por una parte, las carreras pedagógicas poseen un impacto superior en la sociedad chilena, al liderar los cambios en la educación parvularia, básica y media. Por otra parte, la innovación educativa desarrolla el objeto de estudio de las carreras de pedagogía y debería «abordar las dificultades asociadas a la enseńanza y los logros de aprendizaje» (Pey y Chauriye, 2011, p. 60).

Considerando la relevancia adquirida en los últimos años, parece ser relevante explorar la apropiación de académicas y académicos referente al concepto y práctica de la innovación educativa en contexto universitario de carreras pedagógicas.

Para ello, el presente trabajo propone indagar en el estado y características particulares del proceso de innovación educativa en la FID de una universidad estatal (Universidad de Los Lagos) con especial atención a la innovación en el 
área de la docencia. Para ello, se realizará una revisión del concepto de innovación y los criterios que la delimitan. Posteriormente, se realiza una revisión de las experiencias de innovación en las carreras pedagógicas, para concluir con la descripción de las particularidades del derrotero de innovación en la Universidad.

La Universidad de Los Lagos (ULA) es una institución estatal regional, situada en la Décima Región de Los Lagos, al sur de Chile. Las carreras pedagógicas ocupan un lugar importante en la institución, constituyéndose en la principal formadora de docentes en la Región. Actualmente, imparte ocho carreras FID en los diferentes campus, que concentran alrededor de 1350 estudiantes. La importancia de la innovación al interior de las aulas de carreras pedagógicas, se corresponde con los esfuerzos realizados por elevar la calidad profesional de los egresados. Es necesario considerar que, en el caso de las pedagogías, la formación inicial debe intentar erradicar las representaciones y creencias que los profesores en formación traen de sus doce años de escolaridad previa. Estudios demuestran que existe una tendencia en los profesores a desarrollar prácticas de enseñanza muy parecidas a aquellas con las que ellos aprendieron, y que están en directa relación con la concepción de ser docente que han construido a lo largo de su vida (Bruner, 1996; Pozo, 2008; Pozo, 2009; Pozo, 2014, Russell, 2014). La experiencia universitaria debe lograr impactar en las representaciones con que ingresan los estudiantes y la innovación educativa adquiere un carácter urgente al interior de los procesos formativos de la educación superior.

En el año 2010, la universidad implementó un Modelo Educativo Institucional basado en competencias que ha sido el propulsor de la innovación curricular y docente. $\mathrm{Al}$ año 2018, con la mayoría de sus itinerarios formativos en el modelo de formación basado en competencias, la ULA se encuentra abocada a ajustar las prácticas docentes -didácticas y evaluativasal modelo institucional. Para ello, una serie de iniciativas financiadas por el Ministerio de Educación se han realizado para favorecer la armonización de las prácticas docentes al modelo educativo.

\section{Marco teórico}

Blanco y Messina (2000) reconocen en la idea de innovación un enorme grado de relatividad, debido a que se ve influenciada por concepciones ideológicas y epistemológicas acerca del proceso de enseñanza aprendizaje y acerca de las innovaciones educativas mismas. Esto se une a las diferencias contextuales y culturales en las que estos procesos se desarrollan. Unesco (2016) define innovación educativa como: 
Un acto deliberado y planificado de solución de problemas, que apunta a lograr mayor calidad en los aprendizajes de los estudiantes, superando el paradigma tradicional. Implica trascender el conocimiento academicista y pasar del aprendizaje pasivo del estudiante a una concepción donde el aprendizaje es interacción y se construye entre todos (p. 3).

Para Jerez y Silva (2017), la innovación «se entiende como aquel proceso intencionado y permanente al interior de la institución educativa, que pretende provocar transformaciones e impactos reales y positivos sobre: los aprendizajes de los estudiantes, el entorno y cultura institucional y la sociedad» (p. 7).

Ortega, Ramírez, Torres, López, Yacapantli, Suárez y Ruiz (2007), al definir innovación, refieren a «una situación original y otra, distinta, que será el resultado de la innovación» (p. 150). Este cambio implica una interiorización personal de una nueva forma de proceder, pero, además, la institucionalización de esa nueva manera de actuar. Como proceso de institucionalización, la innovación también se desarrolla en múltiples etapas, a través de las cuales las organizaciones transforman paulatinamente sus ideas para mejorar la calidad de todo el sistema (RIE, 2018).

López y Heredia (2017) refieren a innovación educativa como la «implementación de un cambio significativo en el proceso de enseñanza y aprendizaje, de los materiales empleados para el mismo, de los métodos de entrega de las sesiones, de los contenidos o de los contextos que implican la enseńanza» (p. 18). Sin embargo, innovación no necesariamente es igual a novedad (Moreno, 2000). La novedad corresponde a modificaciones superficiales o adopción de algún elemento novedoso, sin que ello constituya una ruptura con la tradición. La innovación educativa, en cambio, desde cualquiera de sus enfoques y en cualquiera de sus niveles, genera modificaciones materiales y simbólicas que permanecen en la cultura institucional. Siguiendo a la misma autora, algunas características relevantes de la innovación educativa son (Moreno, 2000, pp. 24-25):

- La innovación no solo implica algo nuevo, sino también prácticas utilizadas anteriormente, aplicadas a nuevas circunstancias o incorporando nuevos elementos. Esto viene a reforzar la idea que la innovación nunca parte de cero.

- La innovación no es un acto sino un proceso, que involucra personas, situaciones e instituciones, articuladas para generar acciones estructuradas y orientadas a producir cambios.

- La innovación educativa se sustenta en ideas, pero debe reflejarse en prácticas educativas que mejoran la enseñanza aprendizaje. 
El eje central de la innovación educativa es el aprendizaje de los estudiantes y la transición desde un modelo receptivo pasivo de apropiación de conocimientos, a uno activo, socio constructivista de enseńanza aprendizaje. Los profundos cambios sociales que afectan la docencia demandan de una reformulación del modelo de interacción docente-discente y la adaptación de los procesos a los nuevos paradigmas educativos (Coterón y Gil, 2015). El Foro Internacional de Innovación Universitaria (FIIU) define tres ejes de innovación en el contexto universitario (Villa, 2012; 2014; 2016)

- Innovación curricular y pedagógica: Referente a modelos educativos, marcos curriculares, experiencias y metodologías que se requieren para la formación de profesionales en el contexto de la sociedad del conocimiento.

- Innovación social: Referente a la función de la universidad en el contexto actual y su compromiso social.

- Innovación en investigación y tecnología: Referente a las temáticas de investigación relevantes para la sociedad de hoy y el impacto e inserción de la tecnología en la universidad ${ }^{1}$.

$\mathrm{Al}$ interior del primer eje pueden desprenderse dos líneas identificables:

- Innovación curricular: Refiere a la decisión institucional de renovar los planes de estudio y el modelo de docencia asociados, acorde a las demandas del contexto económico, social y cognitivo. La innovación curricular en las universidades latinoamericanas, a partir de la década de 1990, se inspiran en el Proyecto Tuning América Latina. Sus principales acciones son la propuesta de un currículum organizado en un sistema de créditos; el desarrollo de competencias en función de un Perfil de Egreso, que cambia de acuerdo a las necesidades de la sociedad; la incorporación de competencias genéricas y específicas para cada nivel y asignatura (Pey y Chauriye, 2011).

- Innovación de la docencia (pedagógica): Refiere a experiencias metodológicas que emergen en el campo pedagógico del trabajo docente individual, o en conjunto con otros académicos o redes educativas.

1 El FIIU es una asociación sin ánimo de lucro, que funciona en la Universidad de Deusto y presidida por Aurelio Villa, cuyo propósito es constituirse en una plataforma de encuentro de profesionales y entidades vinculadas con la innovación en el ámbito universitario. Estos tres ejes mencionados han sido orientadores del trabajo realizado por la entidad. En el ańo 2018, el FIIU incorpora un cuarto eje de trabajo: Gestión para la innovación, dirigida a aspectos institucionales que posibilitan la innovación. 
La autocrítica reflexiva acerca de las propias prácticas de enseñanza aprendizaje, alimenta propuestas y experiencias que son sistematizadas para producir saber pedagógico. Esta línea releva el rol de las y los docentes como actores del cambio educativo, ya que estas iniciativas están principalmente lideradas por ellos y su capacidad de intervenir creativamente ante problemas pedagógicos.

Jerez y Silva (2017) proponen clasificar la innovación educativa, dependiendo de su nivel de alcance y espacio de acción, en innovación a pequeña escala de las rutinas docentes e innovación disruptiva.

En la innovación a pequeña escala, la acción modifica rutinas claves de profesores, estudiantes y directivos. La disruptiva rompe abruptamente con las rutinas formativas requiriendo procesos de adaptación de la comunidad institucional. La innovación curricular que impacta sobre programas y planes de estudio, de acuerdo a las necesidades emergentes del contexto social institucional, es una innovación disruptiva, ya que obedece a modelos educativos suscritos y las transformaciones se evidencian a mediano y largo plazo. La innovación a pequeña escala, en cambio, refiere a la innovación de la docencia, en la que iniciativas de modificación de rutinas docentes generan cambios acotados, pero de alto impacto y a corto plazo. Al depender su realización de un grupo pequeño de actores, su estabilidad en el tiempo puede verse afectada, ya que no siempre logran instalarse en la institucionalidad (Jerez y Silva, 2017).

Tabla 1. Cuadro comparativo. Tipos de innovación

\begin{tabular}{ll}
\hline \multicolumn{1}{c}{ Innovación a pequeńa escala } & \multicolumn{1}{c}{ Innovación disruptiva } \\
\hline $\begin{array}{l}\text { Genera cambios acotados pero de alto impacto } \\
\text { Impacta en rutinas específicas docentes, de } \\
\text { estudiantes o de la organización }\end{array}$ & $\begin{array}{l}\text { Genera cambios radicales } \\
\text { Impacta en aspectos del proceso } \\
\text { formativo } \\
\text { Bajo nivel de riesgo }\end{array}$ \\
$\begin{array}{l}\text { Nivel de riesgo medio-alto } \\
\text { Implementación progresiva }\end{array}$ & $\begin{array}{l}\text { Implementación por fracturas } \\
\text { Tiempo de instalación e implementación breve de un periodo extenso para } \\
\text { su instalación e implementación. }\end{array}$ \\
$\begin{array}{l}\text { Alto grado de apropiación de un número } \\
\text { mayor de involucrados }\end{array}$ & $\begin{array}{l}\text { Alto grado de apropiación, de un } \\
\text { número pequeńo de involucrados }\end{array}$ \\
$\begin{array}{l}\text { Ejemplo: implementar ciertas rutinas como } \\
\text { plantear preguntas detonantes al inicio de la }\end{array}$ & $\begin{array}{l}\text { Ejemplo: Integrar la metodología } \\
\text { Aprendizaje + Servicio al curso }\end{array}$ \\
\hline clase & \\
\hline
\end{tabular}

Fuente: «Innovando en la Educación Superior» (Jerez y Silva, 2017, p. 18). 
La bibliografía consultada permite advertir que la innovación es caracterizada desde dos dimensiones. Por una parte, existe un énfasis en criterios asociados a la gestión institucional colaborativa de las acciones de innovación. Por otra parte, se relevan los criterios asociados a la gestión de una docencia innovadora en función de metodologías activo participativas.

\subsection{Innovación y gestión institucional}

Desde la perspectiva de la gestión institucional, la innovación es un proceso cimentado en la interacción grupal, el debate de sus miembros, el diálogo y la negociación de posiciones (Unesco, 2016). El carácter innovador está siempre situado en un contexto espacial e histórico de una realidad educativa determinada. Si bien tiene un profundo sentido de cambio y transformación, nunca parte de cero, y se fundamenta en la experiencia previa y la reflexión crítica de los diferentes miembros institucionales. Lo que puede ser innovador en determinada institución, puede no serlo en otra.

Existe consenso en identificar el potencial de una cultura innovadora, la cual facilita que en determinadas instituciones se realicen transformaciones. La presencia de una cultura innovadora depende de las estructuras y procesos micropolíticos de una institución, como el liderazgo facilitador de los directivos y la participación y autonomía de los docentes (Blase, 2002).

Actualmente, se reconocen dos modelos de innovación universitaria, centrados en la gestión institucional:

- Modelo MIES (Modelo de innovación en la educación superior) de iniciativa autónoma de las universidades: Se fundamenta en la competencia organizativa para transformar la acción universitaria a través de la renovación planificada de procesos. Es el modelo más recurrente en Chile y América Latina. El MIES proviene de un modelo propuesto por Villa, Escotet y Goñi (2007). Se basa en cinco dimensiones: entorno y sociedad, estructuras organizativas, infraestructuras, tecnología y recursos, personas y competencias. Estos cinco componentes se entrelazan para la gestión de procesos innovadores (Acosta, Loaíza, Salazar y Salomé, 2016, p. 67).

- Modelo ISUR (Innovación social universitaria responsable): Se fundamenta en el sentido social de la actividad universitaria. Emerge de las necesidades del contexto y de las acciones planificadas de la universidad en sus cuatro ejes (docencia, investigación, gestión, y vinculación con el medio) para impactar positivamente en el contexto social y ambiental. La concepción de este modelo se enmarca en los aspectos misionales 
de docencia, investigación, extensión, servicio social y administración, conjugados en un accionar sinérgico (Villa, 2013).

Como es posible advertir, la innovación es organizada, planificada y sostenida en el tiempo. Es multidimensional, ya que tiene diferentes niveles de concreción, que, en conjunto, permiten la transformación y reorganización de una institución.

\section{Tabla 2. Dimensión Gestión institucional}

\section{Criterios para definir la innovación}

Es un proceso de alcance institucional

Establece un quiebre con la gestión de procesos educativos anteriores

Es planificada y sostenida en el tiempo

Abarca e integra un amplio espectro de procesos, desde la docencia, la vinculación con el medio, la investigación, gestión y otros.

Se desarrolla en colaboración asociativa entre directivos, profesores, estudiantes o redes institucionales externas.

Genera productos para su socialización en la institución: material didáctico, proyectos de investigación, artículos académicos u otros.

Fuente: Elaboración propia, basada en revisión de literatura del área.

\subsection{Innovación y gestión de la docencia}

La gestión de la docencia en los procesos de innovación, obedece a una visión crítica de las prácticas establecidas y la inquietud de mejorarlas. Más que un cambio de paradigmas, es una perspectiva docente, que no concibe la didáctica como un modelo estable, sino como constante revisión y búsqueda de nuevas soluciones a problemas pedagógicos.

La innovación docente tensiona el hábitus pedagógico, generando nuevas prácticas que impactan no solo en el aprendizaje, sino en la cultura institucional completa.

Es recurrente advertir en la gestión de la docencia el fenómeno de la innovación silenciosa, o experiencias innovadoras, con bajo grado de sistematicidad, que no son objeto de análisis, estudios o publicaciones.

Jerez y Silva (2017) reconoce diferentes niveles de impacto en las prácticas innovadoras. Para ello, propone una taxonomía para la evaluación de innovaciones en procesos de enseñanza y aprendizaje en contextos terciarios en tres niveles: 
- Nivel 1: Resultados de aprendizaje que puede lograr una innovación de la docencia.

- Nivel 2: Percepción positiva de los actores vinculados al proceso innovativo. Esto implica motivación de los estudiantes, significatividad asociada al aprendizaje, utilidad de la innovación, entre otros.

- Nivel 3: Impacto en la cotidianidad formativa. Este impacto se refiere a tres focos:

- Rutinas claves: Impacto en las acciones de docentes y estudiantes al interior de procesos de enseñanza aprendizaje.

- Cambios formales: Cambio en los recursos, herramientas y estructuras asociadas a los procesos de enseñanza aprendizaje.

- Uso de un lenguaje pedagógico: Uso del lenguaje para establecer significados, adopción de conceptos claves que pasan a ser propios de la comunidad académica.

Tabla 3. Dimensión Gestión de la Docencia

\section{Criterios para definir innovación}

Responde a una identificada necesidad de mejorar los aprendizajes de los estudiantes. Impacta positivamente en el aprendizaje de los estudiantes.

Desarrolla competencias disciplinares y transversales de manera integrada.

Trasciende al aula y explora otros espacios que no son tradicionales para la formación académica.

Integra la evaluación a la experiencia, con énfasis en evaluación formativa.

Motiva a los estudiantes hacia el aprendizaje.

Se desarrolla en un contexto significativo regional e intercultural.

El estudiante desempeña un rol activo y protagonista.

Considera el aprendizaje colaborativo.

Garantiza el registro y sistematización para el seguimiento del logro de aprendizajes de los estudiantes.

Fuente: Elaboración propia, basada en revisión de literatura del área

\subsection{Innovación educativa y FID}

La innovación educativa, en el contexto de la formación inicial docente, presenta particularidades que relevan la importancia de la innovación en el contexto universitario pedagógico. 
Los estudiantes de pedagogía, frecuentemente, acceden a la formación universitaria provenientes de una cultura escolar pasiva y jerárquica. Los doce años de escolaridad facilitan la naturalización y apropiación de un modelo pedagógico determinante de las prácticas del futuro profesor. El modelo universitario de clase magistral suele reforzar las prácticas tradicionales de los estudiantes de pedagogía, generando una paradoja entre teoría constructivista y prácticas docentes reales (Messina, 1999; Robalino, 2005; Unesco, 2006). El formador de formadores enfrenta la tarea de quebrar las representaciones erigidas en la trayectoria escolar, convirtiéndose en referente del modelo de experiencias de aprendizaje activo-participativas del futuro profesor.

Por otra parte, existe, en la formación inicial docente, algún grado de desvinculación entre la formación disciplinaria y la formación pedagógica. La OCDE (2009) lo advierte y recomienda "promover una mayor colaboración entre las facultades de educación y las otras asignaturas en las universidades» (p. 205). Es relevante, en este contexto, definir como una particularidad de la experiencia de aprendizaje en la formación inicial de profesores, conjugar competencias disciplinarias y pedagógicas, en un ciclo constante de aprender para enseñar.

Moreno (2000) considera además la importancia de formar sujetos innovadores en las carreras de pedagogía, desarrollando habilidades para intervenir de manera creativa en los contextos educativos. Por tanto, a los criterios antes descritos, en las carreras pedagógicas se deben considerar:

Tabla 4. Dimensión Formación Inicial Docente

Criterios para definir innovación

La innovación dialoga con el sistema escolar, a través de prácticas en contextos reales.

Equilibra la formación disciplinaria y la formación pedagógica.

Estimula la metacognición, y posiciona al estudiante en un constante aprender para enseñar.

Fuente: Elaboración propia, basada en revisión de literatura del área.

\section{Metodología}

Para enfrentar el diagnóstico de la innovación en carreras pedagógicas, se realizó trabajo de campo y revisión documental. Se revisaron experiencias docentes para definir el derrotero común de las carreras de pedagogía de la Universidad de Los Lagos hacia la innovación educativa. 
En primera instancia, se realizaron entrevistas a académicos con cargo directivo (jefes de carrera) vinculados a la Formación Inicial Docente. La información fue triangulada con un grupo focal de siete académicos de carreras FID, sin cargo directivo. En un segundo paso metodológico, la revisión documental consideró:

- Informes de autoevaluación de carreras: Realizados entre los años 2013 y 2017, por los comités de autoevaluación de cada carrera, apoyados por la Unidad de aseguramiento de la calidad de la Universidad. El objetivo de estos informes es la revisión crítica de los procesos internos, para presentarse ante el Consejo Nacional de Acreditación, en el correspondiente proceso de acreditación.

- Modelo educativo institucional: Documento que define lineamientos institucionales y declara la formación curricular basada en competencias.

- Diagnóstico de la Formación Inicial de Profesores en la Universidad de Los Lagos: Informe interno levantado por Convenio Marco establecido entre el Ministerio de Educación y la Universidad de Los Lagos, para el diseño del plan de mejoramiento de la calidad de la FID.

La organización de la información se realizó en función de dos categorías previamente establecidas:

1. Concepto de innovación

a. Qué se entiende por innovación

b. Acciones de innovación

c. Actores de la innovación

2. Percepción del estado de la innovación en la institución:

a. Factores que potencian la innovación

b. Factores que dificultan la innovación

\section{Resultados}

\subsection{Concepto de innovación}

Al referirse a la innovación, los académicos transitan conceptualmente entre innovación, novedad, e investigación en procesos pedagógicos, utilizando los tres términos alternadamente y con el mismo fin. Se advierten modelamientos teóricos del concepto, pero no hay referentes comunes. En una misma entrevista 
es posible advertir que los académicos enfatizan en la novedad de la experiencia. Así como en otros momentos, enfatizan en determinantes teóricos de la innovación educativa como, por ejemplo, el impacto logrado sobre el aprendizaje de los estudiantes. Los académicos que demuestran mayor apropiación conceptual coligan innovación e investigación. En esta categoría, es posible advertir coincidencia con la definición de Blanco y Messina (2000), en cuanto al amplio grado de relatividad que alcanza la innovación educativa como concepto.

\subsection{Acciones de innovación}

A través de las entrevistas realizadas a los académicos pertenecientes a la FID y los informes de autoevaluación de carreras revisados, es posible establecer dos ámbitos en los que se realizan iniciativas de innovación: la experimentación con métodos didácticos o evaluativos innovadores y la solución de problemas o debilidades advertidas en el desarrollo de competencias por parte de los estudiantes. La diferencia es importante, en tanto su centro de atención es diferente. El primer grupo focaliza la atención en la metodología y el proceder del profesor; y el segundo, la atención en una dificultad advertida, es decir, en los estudiantes.

Dentro del primer ámbito, las acciones de innovación declaradas en los informes consultados, se asocian a micro investigaciones sobre experiencias de implementación de metodologías didácticas y evaluativas en el contexto de la formulación de un currículum basado en competencias. Las iniciativas proponen integrar metodologías poco frecuentes en su contexto, con énfasis en lo novedoso del diseño de clase (por ejemplo, aprendizaje basado en problemas, metodología de proyectos, entre otras).

En el segundo ámbito, de experiencias orientadas a solucionar dificultades en el desarrollo de determinadas competencias por parte de los estudiantes, las micro investigaciones pesquisadas abordan problemas transversales a la formación de pregrado, como la necesidad de estimular el pensamiento reflexivo, la escritura académica y la incorporación de tecnologías al aula. Si bien, las metodologías con las que se abordan estas dificultades pueden parecer poco novedosas, el énfasis de la iniciativa está en la adaptación de la clase a las necesidades de los estudiantes.

Existe en la FID de la Universidad de Los Lagos una cantidad relevante de experiencias sin sistematizar. Estas pertenecen en su totalidad al ámbito de la implementación de metodologías didácticas y evaluativas para un currículum basado en competencias, y surgen como efecto de una iniciativa individual de algún profesor, que en determinada asignatura desarrolla una experiencia de innovación silenciosa. 
Junto con distinguir dos ámbitos de ejercicio de la innovación (implementación de metodologías didácticas y evaluativas innovadoras e iniciativas para solucionar debilidades en el desarrollo de competencias en los estudiantes), es posible también distinguir dos formas de agenciar las experiencias de innovación. La primera de ellas es, de manera formal, a través de proyectos internos o externos, que habitualmente se socializan fuera de la institución, mediante exposiciones a congresos o publicación de artículos asociados a los resultados de la experiencia. La segunda forma de agenciar experiencias de innovación es de manera informal, espontánea y liderada por el profesor de la asignatura. Esta, en su mayoría, no sale de la institución ni deja productos asociados.

De las entrevistas y la documentación revisada, se identificaron veintiséis experiencias de los últimos cinco años. Solo doce de ellas traspasaron los límites institucionales proyectándose en un artículo académico o presentación en congreso. Catorce no presentan ningún nivel de sistematización.

Tabla 7. Resumen experiencias de Innovación en FID Ulagos

\begin{tabular}{cccc}
\hline $\begin{array}{c}\text { Número de } \\
\text { experiencias } \\
\begin{array}{c}\text { de innovación } \\
\text { en FID }\end{array}\end{array}$ & $\begin{array}{c}\text { Número de académicos } \\
\text { involucrados en } \\
\text { experiencias de } \\
\text { innovación en FID }\end{array}$ & $\begin{array}{c}\text { Número de experiencias } \\
\text { con algún grado de } \\
\text { sistematización (artículos } \\
\text { opresentación a congresos) }\end{array}$ & $\begin{array}{c}\text { Número de } \\
\text { experiencias sin } \\
\text { sistematización }\end{array}$ \\
\hline 26 & 31 & 12 & 14 \\
\hline
\end{tabular}

De la tabla anterior, también es posible inferir el bajo grado de asociatividad entre académicos (treinta y un académicos en veintiséis experiencias), lo que es habitual en un espacio en el que predomina la innovación silenciosa.

Dentro de la primera forma de agenciar las experiencias de innovación, predominan los concursos o fondos internos institucionales. Entre estos, es habitual advertir un ciclo desarrollado por los proyectos: (1) propuestaejecución (2) difusión (3) extinción. Esto significa que en (1) se genera una propuesta hecha por un docente o un grupo de ellos que presentan una innovación en determinada actividad curricular. Con fondos de proyectos o apoyo de las carreras, ejecutan las acciones metodológicas correspondientes a la innovación. (2) Posteriormente, se difunden sus resultados, generalmente, a través de una publicación académica o presentación en congreso. (3) Posterior a la difusión, la innovación no se desarrolla hacia otros ámbitos como la investigación o implementación en contextos más amplios. De esta manera, la iniciativa se extingue en la publicación, cortando el ciclo de desarrollo de la innovación. 


\subsection{Actores de la innovación}

Los académicos sin cargos directivos asocian la capacidad de innovación a ciertas características personales de los académicos (como, por ejemplo, ser «inquietos»; "busquillas»; "preocupados de sus clases»). Los académicos con cargos directivos la asocian principalmente al factor etario («los docentes más jóvenes son más innovadores»).

Se advierte desconocimiento del trabajo entre pares. Esto viene a reforzar la idea de la innovación silenciosa como iniciativa personal.

Los académicos que desarrollan o han desarrollado proyectos de innovación, en el relato de su gestación, mencionan "deseos de cambiar» 0 "romper la rutina». Otra motivación para implementar experiencias de innovación educativa es alinear las experiencias del aula al modelo educativo institucional basado en competencias. Por último, se consideran como una motivación para innovar dificultades que presentan algunos estudiantes para el desarrollo de competencias transversales.

Al revisar la documentación por carreras, es posible advertir que la mayoría de las experiencias de innovación, que traspasa los límites de la universidad con artículos académicos o exposiciones en congresos, es gestionada por académicos con cargos directivos.

\subsection{Percepción del estado de la innovación en la institución}

Entre los entrevistados, se advierte implícito consenso en considerar la docencia como el eje fundamental de la innovación educativa. No reconocen responsabilidades institucionales. Sin embargo, coinciden en que la implementación de experiencias de innovación se inicia con los procesos de ajuste curricular propiciados por el Modelo Educativo Institucional basado en competencias. Las carreras pedagógicas que iniciaron su reformulación curricular se han impuesto desafíos metodológicos y evaluativos que evidencian la necesidad de erigir innovaciones docentes.

Dentro de las fortalezas institucionales para favorecer la innovación en la FID se mencionan (1) concursos de innovación internos desarrollados en los últimos años, y (2) cursos, asesorías y actividades de formación continua desarrollados en la Universidad, que actualizan conocimientos en didáctica y evaluación.

Las debilidades institucionales mencionadas por los académicos como impedimentos para potenciar la innovación educativa son la rotación académica que no permite proyectar procesos a largo plazo y la sobrecarga de trabajo 
administrativo (especialmente en académicos con cargos directivos), y que no permite disponer de tiempo para implementar experiencias de innovación.

\section{DisCUSIÓN Y CONCLUSIONES}

Considerando referentes de innovación establecidos en la literatura del área, se advierte que la innovación educativa en contexto universitario se remite al ámbito de la innovación curricular y pedagógica. Dentro de este marco, predominan experiencias a pequeña escala, que cumplen con los criterios asociados a la dimensión gestión de la docencia y con los criterios asociados a la dimensión FID. Sin embargo, los criterios asociados a la dimensión gestión institucional son los más débiles, efecto de la excesiva personalización de las experiencias de innovación.

El siguiente diagrama representa el derrotero de las iniciativas innovadoras. En este se advierte que el canal de ingreso a la innovación es el modelo educativo por competencias; transita por la innovación curricular y la innovación docente. La innovación docente desarrolla un ciclo al que se le da salida a través de un producto (material educativo o publicación académica) que pone fin al derrotero (representado en sombreado). Se representa también las posibles continuidades a la que aspiran en el área de investigación, considerando la salida ideal, la constitución de una línea de investigación educativa.

La educación, en contexto universitario, hoy se orienta hacia la adopción de una postura reflexiva del ejercicio profesional, que entienda al profesor como un investigador de su propia enseñanza (Chocarro, Sobrino y GonzálezTorres, 2013). La innovación de la docencia se orienta hacia ese modelo profesional, sin embargo, no lo garantiza. Como es posible advertir en la revisión realizada, muchas iniciativas de innovación no logran el desarrollo o consolidación que permite una enseñanza basada en la investigación sobre la misma. Lo anterior permite establecer desafíos institucionales como, por ejemplo, fortalecer la innovación como vía hacia la investigación, aspirando a consolidar líneas acordes a las necesidades advertidas por la educación superior en FID.

La innovación educativa en la Formación Inicial Docente, más que un concepto de apropiación teórica, es una práctica, que se materializa a través de la fuerza docente, en búsqueda de soluciones y nuevas formas de enriquecer el aula de pregrado. 
Figura 1. Derrotero de Innovación educativa desarrollado por las carreras FID en la Universidad de Los Lagos

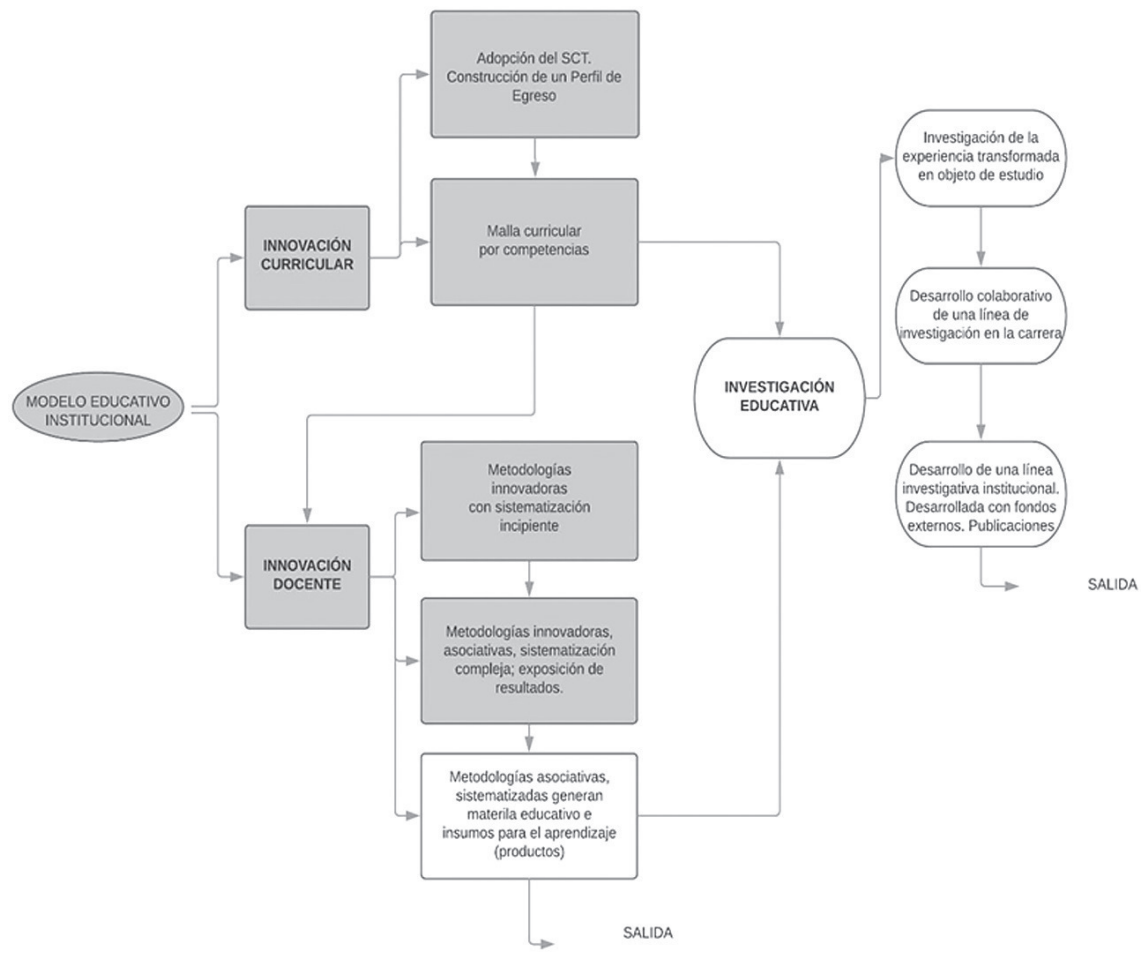

Respecto a las diferentes categorías analizadas y descritas anteriormente, es posible establecer un modelo de innovación presente en la cultura institucional. Este modelo se caracteriza por tres puntos:

- Privilegiar la iniciativa individual, ya que la mayoría de las acciones innovadoras son experiencias docentes particulares.

- Delegar la responsabilidad de la innovación casi exclusivamente al profesor, invisibilizando el entorno institucional que favorece o limita la innovación.

- Desarrollar un ciclo cerrado, ya que la mayoría de las innovaciones se extingue en la publicación académica.

Establecer un trabajo conjunto entre académicos que permita aprehender un concepto de innovación compartido por todas y todos es una urgencia al enfrentar la demanda de innovar en las aulas universitarias. Por otra parte, 
es relevante proyectarse a abrir la cultura innovadora hacia la comunidad, más allá de las experiencias docentes de aula. Concebir la innovación como una oportunidad de explorar la responsabilidad de la academia hacia el contexto en el que está inserta. El desafío es transitar desde el modelo actual basado en la docencia, hacia uno de responsabilidad social, que se haga cargo del carácter regional declarado en el MEI y sus fundamentos socioculturales.

\section{REFERENCIAS BIBLIOGRÁFICAS}

Acosta, S., Loaiza, M., Salazar, A., Salomé, P. (2016). Valoración de la capacidad innovadora de la UTPL a través de la aplicación del Modelo de Innovación en Educación Superior MIES. En A. Villa (ed.), Innovación en la formación del profesorado universitario. España: FIIU.

Blanco, R. y Messina, G. (2000). Estado del arte sobre las innovaciones educativas en América Latina, Santiago de Chile: Unesco. Recuperado de http:// www.observatorioeducativo.pe/wp-content/uploads/2013/11/ESTADODEL-ARTE-SOBRE-LAS-INNOVACIONES-EDUCATIVAS-ENAM\%C3\%89RICA-LATINA.pdf

Blase, J. (2002). Las micropolíticas del cambio educativo. Revista Profesorado, 6, 1-2. Recuperado de http://www.ugr.es/ -recfpro/rev61ART2.pdf

Bruner, J. (1996) The Culture of Education; Cambridge, MA: Harvard University Press.

Coterón, J. y Gil, J. (2015). Innovación educativa en educación secundaria-universidad. Marco teórico y fundamentos para el diseño de proyectos. En T. González, P. Irureta-Goyena y R. Pardo (eds.), La educación experiencial como innovación educativa (pp. 97-124). Madrid: Plaza y Valdés.

Chocarro, E., Sobrino, A. y González-Torres, M.C. (2013). Scholarship of Teaching and Learning: un modelo de desarrollo profesional de los profesores universitarios. Revista Electrónica Interuniversitaria de Formación del Profesorado, 16(1), 5-14. https://doi.org/10.6018/reifop.16.1.179401

Jerez, O. y Silva C. (eds.) (2017). Innovando en Educación Superior: Experiencias clave en Latinoamérica y el Caribe 2016-2017, 1, Gestión curricular y desarrollo de la docencia. Santiago, Chile: Facultad de Economía y Negocios, Universidad de Chile.

López, C. S, Heredia, Y. (2017). Marco de referencia para la evaluación de proyectos de innovación educativa. Guía de aplicación. Monterrey, Nuevo León: Tecnológico de Monterey. Recuperado de http://escalai.com/ wp-content/uploads/2018/12/Gui\%CC\%81a-de-aplicacio\%CC\%81nCorregida_2.pdf 
Messina, G. (1999). Investigación en o investigación acerca de la formación docente: un estado del arte en los noventa. Revista Iberoamericana de Educación, 19, 145-207.

Moreno, M. (2000). Formación de docentes para la innovación educativa. Revista Electrónica Sinéctica, 17, 24-32.

OCDE (2017). Educación en Chile. Evaluaciones de políticas nacionales de educación. Santiago de Chile: Fundación SM.

OCDE (2009). La educación superior en Chile. https://doi.org/10.1787/ 9789264054189-es

Ortega, P., Ramírez, M., Torres, J. López, A., Servín, C., Suárez, L., Ruiz, B. (2007). Modelo de innovación educativa. Un marco para la formación y el desarrollo de una cultura de la innovación. Revista Iberoamericana de Educación a Distancia. Recuperado de http://www.redalyc.org/articulo. oa?id=331427206010

Pey, S. y Chauriye, S. (2011). Innovación curricular en las universidades del consejo de rectores 2000-2010. Recuperado de http://sct-chile.consejoderectores.cl/ documentos_WEB/Innovacion_Curricular/2.Informe_INNOVACION_ CURRICULAR.pdf

Pozo, J.L. (2008). El cambio de las concepciones docentes como factor de la revolución educativa. Revista Educación, Comunicación, Tecnología. 3(5), 1-27.

Pozo, J.L. (2009). Adquirir una concepción compleja del conocimiento: creencias epistemológicas y concepciones del aprendizaje. En J.L. Pozo y M.P. Pérez (eds.), Psicología del estudiante universitario: La formación en competencias. (pp. 70-85). Madrid: Morata.

Pozo, J.L. (2014). Psicología del aprendizaje humano. Adquisición de conocimiento y cambio personal. Madrid: Morata.

RIE 360. (2018). Perspectivas de la innovación educativa en universidades de México: Experiencias y reflexiones de la RIE 360. Recuperado de https:// educatic.unam.mx/avisos/perspectivas-innovacion-educativa-en-universidades-mexico.pdf

Robalino, M. (2005).¿Actor o protagonista? Dilemas y responsabilidades sociales de la profesión docente. Revista PRELAC, 1. Unesco/OREALC.

Unesco. (2006). Modelos innovadores en la formación inicial docente. Santiago de Chile: Unesco.

Unesco. (2016). Texto 1: Innovación Educativa. Herramientas de apoyo para el trabajo docente. Recuperado de http://unesdoc.unesco.org/images/0024/ 002470/247005s.pdf 
Russell,T. (2014). La práctica en la formación de profesores: tensiones y posibilidades en la experiencia de aprender a enseñar. Estudios Pedagógicos, 40 (Especial), 223-238. https://doi.org/10.4067/S0718-07052014000200013

Villa, A. (2012). Avances en la innovación universitaria. Bilbao: Mensajero.

Villa, A. (2014). La innovación educativa para transformar la sociedad multicultural. Recuperado de http://www.foroinnovacionuniversitaria.net/foroant/ la-innovacion-educativa-para-transformar-la-sociedad-multiculturalel-papel-de-las-universidades/

Villa, A. (ed.). (2013). ISUR Un modelo de evaluación de Innovación Social Universitaria Responsable. Bilbao: Universidad de Deusto.

Villa, A. (ed.) (2016). Innovación en la formación del profesorado universitario. Recuperado de http://www.foroinnovacionuniversitaria.net/foroant/ innovacion-en-la-formacion-del-profesorado-universitario/

Villa, A., Escotet, M., Goñi, J. (2007). Modelo de innovación para la educación superior. MIES. Bilbao: Universidad de Deusto. 